\title{
LA JUSTICIABILITÉ DU DROIT À L'ENVIRONNEMENT CONSACRÉ PAR LA CHARTE AFRICAINE DES DROITS DE L'HOMME ET DES PEUPLES DE 1981 EN RÉPUBLIQUE DÉMOCRATIQUE DU CONGO
}

\author{
Kennedy KIHANGI BINDU \\ Professeur associé à l'Université Libre des Pays des Grands Lacs en RD du Congo* \\ kenedybindu@yahoo.fr
}

Reçu: 12 décembre 2012 / Accepté: 21 mai 2013

RÉSUMÉ : Plus de deux décennies après l'entrée en vigueur de la Charte Africaine des Droits de l'Homme et des Peuples, la justiciabilité du droit à l'environnement fait encore l’objet de controverses. Le juge congolais est marqué par des hésitations qui l’empêchent de s'engager dans un procès où ce droit est en cause. Cela, malgré la ratification par la République Démocratique du Congo de la Charte Africaine des droits de l’Homme et des Peuples, l'existence d'une jurisprudence en construction sur le plan continental, la constitutionnalisation du droit à l'environnement pouvant faire l'objet d'une action tant individuelle que collective devant les instances judiciaires. Le contentieux environnemental est quasi inexistant devant les juridictions congolaises malgré les violations manifestes de la législation en matière environnementale. La jurisprudence n’a donc pas contribué à la définition notamment de l'étendue des droits environnementaux des citoyens, des régimes de protection de l'environnement, des obligations de l'État, etc. Il est peu probable que les particuliers réclament ce droit avec succès, par voie judiciaire. Une analyse rétrospective et prospective mérite d'être faite pour des fins utiles. Sinon, la Charte et la Constitution de la RDC serviront de «menu » réservé aux seuls acteurs politiques sans impact réel auprès des populations.

\footnotetext{
* Dr Kihangi Bindu K., LLD / University of South Africa - Unisa, LLM (Master of Laws) University of South Africa - Unisa, LLB (Honours Degree / Licence) Université Libre des Pays des Grands Lacs, Professeur des universités : Université Libre des Pays des Grands Lacs/ULPGL DR Congo, Université de Goma/UNIGOM en RD du Congo, International Leadership University, ILU - Burundi, Université Espoir d'Afrique/Burundi ; Coordinateur du Centre de Recherche sur la Démocratie et le Développement en Afrique (CREDDA) et Membre du Conseil Éditorial de la Revue Meritum de la Faculté de Droit de Universidade FUMEC / Universidade FUMEC au Brésil.
} 
RESUM: Més de dos decennis després de l'entrada en vigor de la Carta Africana dels Drets Humans i dels Pobles, la justiciabilitat del dret al medi ambient encara és objecte de controvèrsies. El jutge congolès viu marcat pels dubtes que l'impedeixen de comprometre's en processos vinculats a aquest dret. Això, malgrat que la ratificació per part de la República Democràtica del Congo de la Carta Africana, l'existència d'una jurisprudència en construcció a escala continental i la constitucionalització del dret al medi ambient podrien fonamentar una acció tant individual com col·lectiva davant de les instàncies jurisdiccionals. El contenciós ambiental és gairebé inexistent davant dels tribunals congolesos, malgrat les violacions manifestes de la legislació en la matèria. La jurisprudència no ha contribuït pas a la definició dels drets ambientals dels ciutadans, dels règims de protecció del medi ambient, de les obligacions de l'Estat, etc. És poc probable que els particulars reclamin aquest dret amb èxit a través de la via judicial. Per tot plegat, és útil una anàlisi retrospectiva i prospectiva, a fi i a efecte d’anar més enllà d’un “menú” reservat per als actors polítics i garantir que la Carta i la Constitució tenen un impacte real per a la població.

RESUMEN: Más de dos decenios después de la entrada en vigor de la Carta Africana de los Derechos Humanos i de los Pueblos, la justiciabilidad del derecho al medio ambiente aún es objeto de controversia. El juez congoleño vive marcado por las dudas, que le impiden comprometerse en procesos relacionados con este derecho. Esto, a pesar de que la ratificación por parte de la República Democrática del Congo de la Carta Africana, la existencia de una jurisprudencia en construcción a escala continental y la constitucionalización del derecho al medio ambiente podrían fundamentar una acción tanto individual como colectiva ante las instancias judiciales. El contencioso ambiental es casi inexistente ante los tribunales congoleños, a pesar de las violaciones manifiestas de la legislación en esta materia. La jurisprudencia no ha contribuido a la definición de los derechos ambientales de los ciudadanos, de los regímenes de protección del medio ambiente, de las obligaciones del Estado, etc. Es poco probable que los particulares reclamen este derecho con éxito a través de la vía judicial. Por todo ello, es útil un análisis retrospectivo y prospectivo, con el fin de ir más allá de un "menú” reservado a los actores políticos y garantizar que la Carta y la Constitución tienen un impacto real sobre la población. 
ABSTRACT: More than two decades after the entering into force of the African Charter on Human and People's Rights, the justiciability of the right to a healthy environment is still controversial. Congolese judges are under the shadow of the doubt, being impeded to commit themselves with procedures involving this right, despite the ratification of the Charter by the Democratic Republic of the Congo, the existence of a continental case-law and the constitutionalization of the right to a healthy environment could be the foundation for individual and collective actions before courts. Environmental lawsuits are almost inexistent in Congo, despite massive violations in this field. Case-law hasn't contributed to the definition of environmental rights of citizens, the protection regimes and state's obligations. It is not much probable that individuals claim for this right before courts. For all these reasons, it is useful a retrospective and prospective analysis, aiming to go beyond a void declaration for political purposes and guaranteeing a real impact on the population of the Charter and the Constitution.

MOTS CLÉS : Environnement — Droits humains — Justiciabilité — Charte Africaine des Droits de l'Homme et des Peuples - Constitution.

PARAULES CLAU: Medi ambient — Drets humans - Justiciabilitat — Carta Africana dels Dret Humans i dels Pobles — Constitució.

PALABRAS CLAVE: Medio ambiente - Derechos humanos - Justiciabilidad - Carta Africana de los Derechos Humanos y de los Pueblos — Constitución.

KEYWORDS: Environment — Human rights — Justiciability — African Charter on Human and People’s Rights - Constitution.

Sommaire : I. Introduction. II. Reconnaissance du droit à l'environnement sur les plans universel et régional. 1. Sur le plan universel. 2. Sur le plan régional. III. Obligations des États signataires de la Charte Africaine des droits de l'homme et des peuples dans la protection et la promotion du droit à l'environnement. IV. Le particularisme de la Charte Africaine et de la jurisprudence dans le domaine des droits des deuxième et troisième générations. V. Le droit à l'environnement dans l'ordre juridique congolais. VI. Les juridictions congolaises face à la protection et à la promotion du droit à un environnement sain. 1. Applicabilité de la Charte Africaine des Droits de l'Homme et des Peuples devant les cours et tribunaux congolais. 2. La jurisprudence congolaise en 
matière de droit de l’homme à un environnement sain. VII. Conclusion et recommandations. VIII. Bibliographie finale.

\section{INTRODUCTION}

Plus de deux décennies après l'entrée en vigueur, et la ratification par la République Démocratique du Congo, ${ }^{1}$ de la Charte Africaine des Droits de l'Homme et des Peuples (CADHP) en 1986, ${ }^{2}$ la juridicité des droits de la solidarité ou droits de la troisième génération ${ }^{3}$ mais aussi de ceux de la deuxième génération qu'elle organise, ne rencontre pas encore l'unanimité. Les controverses sont fréquentes dans différents milieux notamment académiques, diplomatiques, politiques ainsi que judiciaires à la fois sur les plans national, régional et international. De la même façon que les droits sociaux, économiques et culturels sont apparus nécessaires pour rendre effectifs les droits civils et politiques, les droits de la solidarité ou droits collectifs seraient la condition d'existence des droits de la première et de la deuxième générations. ${ }^{4}$ Les droits de la solidarité sont non seulement consacrés par la Charte Africaine des Droits de l'Homme et des Peuples ${ }^{5}$ mais aussi par un nombre impressionnant des constitutions africaines notamment celle de la République Démocratique du Congo (RDC). ${ }^{6}$ Il est cependant étonnant de constater que les énergies déployées pour la constitutionnalisation de ces droits ne sont pas mises à profit dans la phase de leurs mises en

\footnotetext{
${ }^{1}$ Ordonnance Loi No 87-027 du 20 Juillet 1987 portant ratification par la RDC de la Charte Africaine des Droits de l'Homme et des Peuples. Lire le Journal Officiel de la République Démocratique du Congo, Instruments juridiques internationaux et régionaux relatifs aux droits de l’homme ratifiés par la République Démocratique du Congo, Cabinet du Président de la République, $43^{\text {ème }}$ numéro, décembre 2002, p. 244.

${ }^{2}$ Aussi appelée la « Charte de Banjul », la Charte Africaine des Droits de l'Homme et des Peuples a été adoptée par l'OUA en juin 1981 et est entrée en vigueur en octobre 1986. Il est reconnu à la Charte la valeur d'un instrument central des droits de l'homme de l'Organisation de l'Unité Africaine (OUA) appelée aujourd'hui Union Africaine (UA). Sélection des documents clés de l'Union Africaine relatifs aux droits de l'homme, Pretoria University Law Press, 2008, p. 21.

${ }^{3}$ Certains doctrinaires à l'instar de Frédéric Sudre qualifient ces droits de «pseudo-droits » et que leur introduction dans la catégorie des droits de l'homme fait perdre à celle-ci toute unité conceptuelle et toute cohérence intellectuelle et menace la notion même de droits de l'homme. Sudre F., Droit international européen des droits de l'homme, $5^{\text {ème }}$ éd., PUF, Paris, 1989, p. 175/189.
}

\footnotetext{
${ }^{4}$ Mazyambo Makengo Kisala A., «Introduction aux droits de l'homme: Théorie générale, instruments, mécanismes de protection» du Programme des Nations Unies pour le Développement, Projet Appui aux institutions de la transition "Mandats, rôle et fonctions des pouvoirs constitués dans le nouveau système politique de la République Démocratique du Congo : Journées d'information et de formation organisées à l'intention des parlementaires, des députés provinciaux et de hauts cadres de l'administration », Kinshasa, 2007, p. 232.

${ }^{5}$ Articles 22 à 24 de la Charte.

${ }^{6}$ Articles 50 à 61 de la Constitution de la RDC telle que modifiée par la Loi n 11/002 du 20 janvier 2011 portant révision de certains articles de la Constitution de la République Démocratique du Congo du 18 février 2006. Il s'agit principalement du droit au développement, droit à la paix et à la sécurité, droit à un environnement sain et droit au patrimoine commun de l'humanité.
} 
œuvre. Une simple observation révèle des attitudes inquiétantes de la part des juges congolais ayant le mandat constitutionnel de garantir les droits et libertés fondamentaux en RDC. ${ }^{7}$ Le droit à l'environnement est particulièrement au centre des controverses et misunderstandings liés à son contenu, ses caractères individuel et collectif : Peut-il bénéficier de la même protection que les autres droits fondamentaux et faire l'objet d'un examen devant les instances judiciaires en RDC ? Il est, certes, peu probable que les particuliers réclament ce droit avec succès, par voie judiciaire, malgré la tendance moniste adoptée par le constituant congolais. Ce monisme ainsi que la constitutionnalisation du droit à l'environnement annonce une obligation constitutionnelle qui pèse sur les cours et tribunaux congolais d'appliquer directement la Charte Africaine des Droits de l'Homme et des Peuples. Contre cet environnement, et pour éclairer la lanterne des uns et des autres, il est judicieux de poser la question de savoir : Sur quelle base les victimes d'une violation du droit à l'environnement en RDC peuvent-elles agir en justice ? Quelles sont les prestations justiciables ? Cela est d'autant plus indispensable en vue de relever l'incidence de la Charte Africaine des Droits de l'Homme et des Peuples (CADHP) sur les décisions judiciaires en RDC ? En effet, la Charte Africaine des Droits de l'Homme et des Peuples reste un outil qui révolutionne et nourrit la connaissance ainsi que la culture du respect, de la protection et de la promotion des droits de l'homme notamment ceux des deuxième et troisième générations en RDC. Il reste néanmoins à faire une analyse rétrospective et prospective pour un avenir garantissant un bien être pour tous. Les peuples doivent être informés de leurs droits, des engagements internationaux ratifiés par la RDC ainsi que des mécanismes de revendication mis en place sur les plans à la fois national, régional et international. La justice doit être reconnue aux peuples non pas comme un acte de charité mais comme un droit. ${ }^{8}$ Dans le cas contraire, la Charte Africaine des Droits et des Peuples ainsi que la Constitution de la RDC seront considérées comme des menus réservés aux seuls acteurs politiques sans impact réel auprès des populations.

\footnotetext{
${ }^{7}$ L'article 150 de la Constitution de la RDC telle que modifiée par la Loi n 11/002 du 20 janvier 2011 portant révision de certains articles de la Constitution de la République Démocratique du Congo du 18 février 2006 prévoit que :

«Le pouvoir judiciaire est le garant des libertés individuelles et des droits fondamentaux des citoyens. Les juges ne sont soumis dans l'exercice de leur fonction qu'à l'autorité de la loi. Une loi organique fixe le statut des magistrats ».

${ }^{8}$ Kihangi Bindu K. Environmental and developmental rights in the Southern African Development Community: Specific reference to the Democratic Republic of Congo and the Republic of South Africa, LAP Lambert Academic Publishing, Saarbucken, Germany, 2011, p. 355.
} 


\section{RECONNAISSANCE DU DROIT À L'ENVIRONNEMENT SUR LES PLANS UNIVERSEL ET RÉGIONAL}

Un nombre d'instruments juridiques sur les plans universel et régional font mention directement ou indirectement du droit de l'homme à l'environnement exprimant ipso facto son originalité et ses spécificités.

\section{Sur le plan universel}

Depuis plusieurs décennies, les doctrinaires ne s’accordent pas sur la reconnaissance et le lien existant entre les droits humains et l'environnement. Miller considère que les droits humains ne peuvent pas être invoqués pour la protection de l'environnement. ${ }^{9}$ Cependant, une évolution récente révèle des acquis significatifs quant à la reconnaissance du droit à l'environnement et le lien existant entre les droits humains et l'environnement. ${ }^{10}$ L'idée de la prise en compte de l'environnement a fait son apparition avec force au travers de la Déclaration Universelle des Droits de l'Homme ${ }^{11}$ qui vise le droit à la vie dans son article $3^{12}$ et le droit à la santé dans son article $25 .{ }^{13}$ À cela, il faut ajouter le Pacte International relatif aux Droits Civils et Politiques qui proclame le droit à la sécurité ${ }^{14}$ ainsi que le Pacte International relatif aux Droits Économiques, Sociaux et Culturels qui consacre aussi le droit à la santé. ${ }^{15}$ Certains auteurs associent également le droit à l’environnement au droit à la vie. ${ }^{16}$

\footnotetext{
${ }^{9}$ Miller C “Environmental rights: Critical perspective”, 1998, in Thornton J. \& Beckwith S., Environmental law, $2^{\text {nd }}$ ed. Sweet and Maxwell Thomson London, 2004, p. 368.

${ }^{10}$ Brown Weiss E., (ed.) Environmental change and international law: New challenges and dimensions, Tokyo, United University Press, 1992, p. 274; Kiss A. and Shelton D., Guide to international environmental law, Martinus Nijhoff Publishers, Leiden/Boston, 2007, p. 237/241.

${ }^{11}$ Déclaration Universelle des Droits de l'Homme du 10 décembre 1948, Journal Officiel du Zaïre/RD Congo, Nº Spécial, avril 1999, p. 7.

12 Article 3 :

« Tout individu a droit à la vie, à la liberté et à la sûreté de sa personne ».

${ }^{13}$ Article 25 :
}

«1. Toute personne a droit à un niveau de vie suffisant pour assurer sa santé, son bien-être et ceux de sa famille, notamment pour l'alimentation, l'habillement, le logement, les soins médicaux ainsi que pour les services sociaux nécessaires ; elle a droit à la sécurité en cas de chômage, de maladie, d'invalidité, de veuvage, de vieillesse ou dans les autres cas de perte de ses moyens de subsistance par suite de circonstances indépendantes de sa volonté.

2. La maternité et l'enfance ont droit à une aide et à une assistance spéciales. Tous les enfants, qu’ils soient nés dans le mariage ou hors mariage, jouissent de la même protection sociale ».

${ }^{14}$ Article 9.

${ }^{15}$ Article 12. 
La Déclaration de Stockholm de 1972 est le document fondateur en droit de l'environnement dont le principe 1 proclame que « l’homme a un droit fondamental à la liberté, à l'égalité et à des conditions de vie satisfaisantes, dans un environnement dont la qualité lui permette de vivre dans la dignité et le bien-être. Il a le devoir solennel de protéger et d'améliorer l’environnement pour les générations présentes et futures ».

En 1990, l’Assemblée générale des Nations Unies a adopté une résolution selon laquelle l'Assemblée : ${ }^{17}$

« Reconnaît que toutes les personnes ont droit à vivre dans un environnement propre à assurer leur santé et leur bien-être ».

Cette résolution confirme l'existence du droit à l'environnement au plan mondial. Elle est proche du texte du Pacte et elle précise que le droit à l'environnement est très lié à la santé de l'homme. Au cours des années 90, la Commission des droits de l'homme a affirmé l'existence de relations inextricables entre droits de l'homme et environnement. ${ }^{18}$ En 1991, elle a adopté une résolution reconnaissant que «tout individu a droit de vivre dans un environnement propre à assurer sa santé et son bien-être ». ${ }^{19}$

La Déclaration de Rio de 1992, quant à elle fait, mention de ce droit au travers de son principe 1 en ces termes: "Les êtres humains sont au centre des préoccupations relatives au développement durable. Ils ont droit à une vie saine et productive en harmonie avec la nature ». Bien que non obligatoires, ces textes ont joué un rôle culminant pour la promotion du droit à l'environnement. Les travaux de la commission des droits de l'homme des Nations Unies, après le rapport de Mme Ksentini du 26 juillet 1994 consacré au droit de l'homme à l'environnement dans le monde, a préparé un projet de déclaration sur les droits de l’homme et de l'environnement qui consacre pour tous un droit à un environnement sain, sûr et

\footnotetext{
${ }^{16}$ Le droit à la vie du Pacte international relatif aux droits civils et politiques (art. 6.1) peut justifier l'interdiction des pollutions graves car il ne se limite pas au droit de ne pas perdre la vie. Au Pakistan, la Cour suprême a considéré que le droit à la vie inclut le droit de vivre dans un environnement sain. Lau, M., p. 285 in Boyle A., \& Anderson M., Human Rights Approaches to Environmental Protection, Clarendon, Oxford, 1998.

${ }^{17}$ United Nations General Assembly, Resolution on the Need to Ensure a Healthy Environment for the Wellbeing of Individuals. A/RES/45/94 (adopted on 14th Dec.1990, 68th Plenary Meeting).

"The General Assembly ... Reaffirming that in accordance with the Declaration of the United Nations Conference on the Human Environment, men and women have the fundamental right to freedom, equality and adequate conditions of life in an environment of quality that permits a life of dignity and well-being...

1. Recognizes that all individuals are entitled to live in an environment adequate for their health and well-being; ...”).

${ }^{18}$ E/CN.4/Sub. 2/1990/59, p. 25 Rés. 1990/7.

${ }^{19}$ E/CN.4/Sub.2/1991/91.
} 
écologiquement rationnel ainsi qu'un droit à une eau et à des aliments sains. ${ }^{20} \mathrm{D}$ 'autres instruments internationaux ont accordé aussi une importance particulière au droit à l'environnement, notamment : la convention sur les droits de l'enfant du 20 novembre 1989 qui impose aux États de protéger la santé des enfants en prenant spécialement en considération les risques causés par la pollution de l'environnement. La Convention 169 de l'Organisation Internationale du Travail (OIT) relative aux peuples indigènes dans des pays indépendants du 27 juin 1989 invite les États à prendre des mesures spéciales pour sauvegarder l'environnement de ces peuples (art. 4.1).

\section{Sur le plan régional}

Le droit de l'homme à l'environnement a connu un progrès juridique impressionnant sur le plan régional. La Charte Africaine des Droits de l’Homme et des Peuples de 1981 est le premier traité international reconnaissant le droit de l'homme à l'environnement. Son article 24 proclame que : «Tous les peuples ont un droit à un environnement satisfaisant et global, propice à leur développement $»^{21}$

À la lecture de cette disposition, sans nul doute, la Charte Africaine des Droits de l'Homme et des Peuples reconnaît le droit à l'environnement au titre d'un droit de l'homme. Cependant, lorsqu'on cherche à savoir à quoi renvoie ce droit et qui en est le titulaire, des controverses surgissent. En effet, pour qualifier l'environnement, selon Hervé Jiatsa, ${ }^{22}$ les rédacteurs de la Charte ont choisi des mots «suffisamment imprécis pour se prêter à toutes sortes d'interprétations » comme satisfaisant, global etc. Mohamed Ali Mekouar ${ }^{23}$ estime qu'on

\footnotetext{
${ }^{20}$ Ksentini Z., Droit de l'Homme et Environnement, Commission des droits de l'Homme, Conseil Économique et Social des Nations Unies, 6 juillet 1994 ; Prieur M., Les principes généraux du droit de l'environnement, notes de cours, $N^{\circ} 5$, Université de Limoges, France, p. 9.

${ }^{21}$ Par cette reconnaissance formelle, l'Afrique avait fait une apparition surprenante en proclamant dans un instrument juridique contraignant ce que le monde entier avait jusque-là accepté seulement dans des textes de " soft Law» comme la Déclaration de Stockholm de 1972 et celle de Rio de 1992. On se serait attendu à ce que l'Afrique qui est un continent en proie au sous-développement et à la pauvreté soit réticente pour une telle consécration et qu'elle mette en avant son droit à un développement économique renvoyant ainsi les questions liées à l'écologie aux pays développés. L'Afrique a fait preuve de réalisme et n'est pas tombée dans ce piège. À travers la charte Africaine des droits de l'homme et des peuples, elle a plutôt adopté une attitude de conciliation et mis dans le même sac le droit à la paix, le droit au développement et le droit à l'environnement. JIATSA Meli H., Les droits fondamentaux et le droit à l'environnement en Afrique, 2007, titre récupéré le $1^{\mathrm{er}}$ octobre 2012 ; disponible sur : <http://www.memoireonline.com/10/10/4025/m_Les-droits-fondamentaux-et-le-droit-lenvironnement-en-Afrique7.html>.

${ }^{22}$ Jiatsa Meli H., op. cit.

${ }^{23}$ Mekouar A. M., Le droit de l'environnement dans la Charte Africaine des droits de l'homme et des peuples, étude juridique de la FAO en ligne \# avril 2001, titre récupéré le 9 octobre 2012, disponible sur : <http : //www.fao.rg/Legal/default.htm>.
} 
aurait pu utiliser des mots plus précis comme «sain, salubre, propre, équilibré, décent, convenable, de qualité, dignité, bien-être, intérêt des générations futures etc. " pour donner plus de sens. La difficulté de cerner le droit à l'environnement dans la Charte Africaine n'est pas spécifique à ce texte. Cela parce que même en France, au sujet de la consécration législative, Michel Prieur reconnait qu’ «il était difficile de formuler concrètement ce droit fondamental nouveau ». ${ }^{24}$ Mohamed Ali Mekouar explique ce flou par le fait que les rédacteurs de la Charte ne s’étaient basés sur aucun précédent dans les instruments internationaux de protection des droits de l'homme car ils faisaient un peu œuvre de pionniers. $^{25}$

L'autre difficulté majeure que connaît cette Charte est liée au titulaire du droit à l'environnement. Il ressort d'une analyse de ce texte qu'il est résolument tourné vers une approche collective puisque allusion est faite au « peuple ». Il convient de préciser que cette approche collective n'est qu'apparente car le droit consacré dans la Charte protège aussi bien le peuple dans son ensemble (droit collectif pouvant être exercé par une association, un groupe ayant la capacité d'intenter en justice) que les personnes prises individuellement (toute personne dont le droit à l'environnement a été violé pouvant demander à ce que justice soit faite). ${ }^{26}$ Le terme «peuple » contenu dans l'article 22 de la Charte mérite d'être compris et défini tout en réservant une place de choix et une conformité à la lettre et à l'esprit de la Charte enracinés dans les valeurs de la civilisation africaine. Le droit à l'environnement est ainsi présenté par la Charte prenant en compte les deux approches individuelle et collective. Cela trouve son explication dans la philosophie africaine de l'existence présentée par Mbiti :

"I am because we are and since we are therefore I am"27.

Ankumah précise que vivre en Afrique signifie :

"Abandoning the right to be an individual, particular, competing, selfish, aggressive, conquering, being in order to be with others, in peace and harmony with the living and the dead, with the natural environment and the spirit that people is or give life to it”,28.

\footnotetext{
${ }^{24}$ Prieur M., Droit international et comparé de l'environnement: Les principes généraux du droit de $\mathrm{L}^{\prime}$ environnement, cours $\mathrm{n}^{\circ}$ 5, formation à distance, campus numérique, Université de limoges, p. 12.

${ }^{25}$ Mekouar A. M., op. cit.

${ }^{26}$ Jiatsa Meli H., op. cit.

${ }^{27}$ Mbiti JS African religions and philosophy, 1970, p. 144 in Ankumah E. A., The African Commission on Human and Peoples' Rights: Practice and procedures, Vol. 16, Martinus Nijhoff Publishers, The Hague, London, Boston, 1996, p. 159.
} 
En effet, si ce droit a pu rester pendant longtemps une abstraction, il put bientôt devenir juridique avec l'entrée en vigueur le 25 janvier 2004 du Protocole de Ouagadougou du 8 Juin 1998 créant la Cour Africaine des Droits de l’Homme et des Peuples. À travers ce texte, la Cour sera en mesure de juger des atteintes à ce droit nouveau et devra surmonter la difficulté d'interprétation liée à la reconnaissance non pas d'un droit individuel mais d'un droit collectif. Pourraient alors en bénéficier les groupes victimes des catastrophes écologiques et les réfugies écologiques. La Cour africaine pourrait être saisie par la Commission africaine des Droits de l'Homme et des Peuples, les États Parties à la Cour et les organisations intergouvernementales africaines. Elle pourra également recevoir, en cas d'épuisement des voies de recours internes, des requêtes formulées par des Organisations Non Gouvernementales (ONG) dotées du statut d'observateur auprès de la Commission africaine, mais aussi d'individus si l'État concerné a donné une autorisation en ce sens en faisant une déclaration au titre de l'article 34 (6) du protocole. ${ }^{29}$

La convention de 1968 sur la consécration de la nature et des ressources naturelles a également constitué l'un des moments forts ayant précédé la consécration du droit à l'environnement par la Charte Africaine des Droits de l'Homme et des Peuples. La Convention de 1968, dans son article 2, venait déjà de mettre à la charge des États le devoir de prendre des mesures nécessaires pour une gestion des ressources naturelles au profit des peuples. La Charte Africaine de l'Organisation de l'Unité Africaine (OUA) obligeait également dans son préambule les États à utiliser les ressources du continent dans l’intérêt des peuples africains. L’Afrique a dès les premières heures voulu démontrer l'importance de placer l’homme au cœur des préoccupations environnementales.

La dynamique environnementale semble avoir atteint la plupart des sous-régions du continent africain qui, dans leurs textes constitutifs, consacrent directement le droit à l'environnement ou y font allusion de manière implicite. À la lecture de ces textes, il se dégage véritablement une prise de conscience environnementale manifeste. Tel est le cas de la Southern African Development Community (SADC) dans son texte constitutif. ${ }^{30}$ Une attention particulière est

\footnotetext{
${ }^{28}$ Keba M. \& Birame N., The Organization of African Unity, the international dimension of human rights, Vol. 2, (1982) , P. 583 in Ankumah E., A., op. cit. 160. In traditional Africa the community's interest was always supreme even though this was thought to be complementary to, rather than in competition with, individual rights. Individual is considered to be 'part and parcel' of a group. The complexity of rights and duties in African societies has been characterized under four Rs: "Respect, Restraint, Responsibility and Reciprocity."

${ }^{29}$ Article 5 du Protocole relatif à la Charte Africaine des Droits de l’Homme et des Peuples portant création d'une Cour africaine des Droits de l'Homme et des Peuples (1998/2004).

${ }^{30}$ Article 5 de l'Acte constitutif de la SADC, Windhoek (Namibie), 17 août 1992 : "The objectives of SADC shall be to: a) achieve development and economic growth, alleviate poverty, enhance the standard and quality of
} 
ainsi attachée à un certain nombre d'éléments, notamment la lutte contre la désertification, les inondations, la déforestation, la pollution, la protection de la diversité biologique et la promotion de l'exploitation rationnelle des ressources naturelles.

En effet, la consécration africaine du droit à l'environnement à travers une procédure juridictionnelle innovante doit être rattachée à un autre événement important du droit international de l'environnement en Afrique. Il s'agit de l'adoption d'une nouvelle Convention Africaine sur la Conservation de la Nature et de Ressources Naturelles à Maputo, le 11 juillet 2003, qui devait entrer en vigueur après le dépôt de 15 instruments de ratification. Les articles 3 et 16 présentent respectivement la reconnaissance d'un droit de l'homme à l'environnement ainsi que la définition des droits procéduraux suivants :

« En prenant des mesures pour réaliser les objectifs de la présente Convention et mettre en œuvre ses dispositions, les Parties seront guidées par :

1. Le droit de tous les peuples à un environnement satisfaisant qui favorise leur développement ;

2. Le devoir des États, individuellement et collectivement, d'assurer l'exercice du droit au développement ;

3. Le devoir des États de veiller à ce que les besoins en matière de développement et d'environnement soient satisfaits de manière durable, juste et équitable ».

«1. Les parties contractantes adoptent les mesures législatives et réglementaires nécessaires pour assurer à temps et de manière appropriée :

a. La diffusion d'informations sur l'environnement ;

b. L'accès du public aux informations sur l'environnement ;

c. La participation du public à la prise des décisions pouvant avoir un impact important sur l'environnement ;

d. L'accès à la justice en ce qui concerne les questions liées à la protection de l'environnement et des ressources naturelles ».

Manifestement, ces dispositions renforcent la Charte Africaine des Droits de l'Homme et des Peuples de 1981, la compréhension du caractère justiciable du droit à l'environnement ainsi que le droit d'avoir accès à la justice et de présenter des revendications liées à la protection de l'environnement. De même, l'Acte constitutif de l’Union Africaine reprend les notions de développement durable et insiste sur les droits des peuples, droits de la troisième génération.

life of the people of Southern Africa and support the socially disadvantaged through regional integration; ...g) achieve sustainable utilization of natural resources and effective protection of the environment ...". 
Ce particularisme s'explique par le contexte africain post-colonialiste où les droits civils et politiques ne peuvent se développer sans la réalisation conjointe des droits socio-économiques et culturels et des droits de la solidarité.

Sur le continent américain, le système de protection des droits humains a une base normative reposant sur plusieurs instruments notamment : la Déclaration américaine des droits et devoirs de l'homme de 1948, la Convention américaine relative aux droits de l'homme adoptée en 1978 et le Protocole additionnel à la Convention américaine relative aux droits de l'homme traitant des droits économiques, sociaux et culturels (Protocole de San Salvador), entrée en vigueur en 1999. La Déclaration américaine reconnaît une série de droits civils, politiques, économiques, sociaux et culturels. La Convention américaine reconnaît une vaste gamme de droits civils et politiques mais ne stipule pas explicitement les droits ESC des personnes sous la juridiction des États parties. Elle inclut toutefois une formule générique qui renvoie aux dispositions des droits ESC de la Charte de l’Organisation des États américains. Elle prescrit ce qui suit au chapitre III, article 26, sous le titre de « Développement progressif » :

«Les États parties s'engagent, tant sur le plan intérieur que par la coopération internationale notamment économique et technique, à prendre des mesures visant à assurer progressivement la pleine jouissance des droits qui découlent des normes économiques et sociales et de celles relatives à l'éducation, la science et la culture, énoncées dans la Charte de l’Organisation des États américains, réformée par le Protocole de Buenos Aires, et ce en fonction des ressources disponibles et par l'adoption de dispositions législatives ou par tous autres moyens appropriés ».

L'article 11 du Protocole additionnel à la Convention américaine relative aux droits de l’homme, traitant des droits économiques, sociaux et culturels, adopté à San Salvador en 1988 et entré en vigueur en 1999, proclame que « Chacun a le droit de vivre dans un environnement sain et de bénéficier des équipements publics essentiels ». Ce texte définit l'obligation des États parties d' « adopter les mesures nécessaires, qu’elles soient de nature interne ou qu'elles relèvent de la coopération entre États, tout particulièrement dans les domaines économique et technique, selon les ressources disponibles et compte tenu de leur degré de développement, pour parvenir progressivement, et conformément à la législation interne, à assurer le plein exercice des droits reconnus dans le présent Protocole ». Visiblement, les concepts contenus dans les expressions « selon les ressources disponibles » et "progressivement » ont été tirés de l'article 2 du Pacte international relatif aux droits économiques, sociaux et culturels, et de l'article 26 de la Convention américaine des droits de l'homme. Le Protocole de San Salvador 
fait nettement avancer les droits ESC qui y sont stipulés par rapport à la manière dont ils figurent dans la Déclaration et la Convention. La teneur des droits et obligations des États y est définie plus précisément. Ce texte fait de ce droit de l'homme un droit individuel. ${ }^{31} \mathrm{Ce}$ texte a été renforcé par l’article 13 du Projet de Déclaration interaméricaine relative aux droits des peuples autochtones : «Les populations indigènes ont droit à un environnement sain, condition essentielle à la jouissance du droit à la vie et au bien-être ». ${ }^{32}$

En Europe, la protection de l'environnement ne figure pas explicitement dans la Convention européenne de sauvegarde des droits de l’homme et des libertés fondamentales, adoptée à Rome en $1950 .^{33}$ La Charte sociale européenne adoptée en 1961 ne traite pas explicitement de la protection de l'environnement. Toutefois, le droit à l'environnement découle en partie du droit à la protection de la santé qui fait partie des articles obligatoires de la Charte sociale pour tous les États de l’Union européenne. Les années 1970 ont été marquées par une forte prise de conscience environnementale. En ce sens, des propositions ont été faites pour compléter la Convention européenne par un Protocole additionnel «garantissant à chacun un droit de jouir d’un environnement sain et non dégradé ». Dans cette dynamique, la Convention d'Aarhus de 1998 sur l'accès à l'information, la participation du public au processus décisionnel et l'accès à la justice en matière d'environnement a rattaché ces droits procéduraux au droit fondamental à l'environnement dans son préambule en énonçant que : « Chacun a le droit de vivre dans un environnement propre à assurer sa santé et son bienêtre ». L’article premier de cette Convention oblige les États à : « Protéger le droit de chacun,

\footnotetext{
${ }^{31}$ Ksentini Z., op. cit.

${ }^{32}$ Texte en discussion devant l’Assemblée Générale de l’Organisation des États américains en 1999.

${ }^{33}$ Retenons cependant que la Cour Européenne des Droits de l'Homme (CEDH) s'est investie dans l'examen des affaires portant sur des atteintes à l'environnement affectant la santé. Ainsi, dans l'affaire Lopez - Ostra (9/2/1994) qui concernait des nuisances par des pollutions et des odeurs, la Cour a déclaré : « Il va de soi que des atteintes graves à l'environnement peuvent affecter le bien-être d'une personne et la priver de la jouissance de son domicile, de manière à nuire à sa vie privée et familiale, sans pour autant mettre en grave danger la santé de l'intéressé ». Manifestement cette phrase essentielle détache le droit à l'environnement du droit à la santé. Dans l'affaire Guerra (19/2/98) où la requête était fondée sur l'absence d'information des populations exposées aux risques d'une usine chimique, la Cour a déclaré : "L'information du public représente désormais l'un des arguments essentiels de la protection, du bien-être et de la santé de la population dans les situations de danger pour l'environnement». Dans l'affaire McGinley et Egan v. Royaume Uni (9/6/1998) et l'affaire LCB v. Royaume-Uni (9/6/1998), la Cour affirme l'obligation de prendre les « mesures nécessaires à la protection de la vie des personnes relevant de sa juridiction ». Il convient également de rappeler l'affaire Zander contre la Suède du 25/11/1993 relative au «droit de jouir de l'eau de leurs puits comme boisson, élément de leur droit de propriétaires du terrain », p. 27. Déjeant-Pons M. : « Le droit de l'homme à l'environnement : Droit fondamental au niveau européen dans le cadre du Conseil de l’Europe », R.J.E., Nº 4, 1994, p. 373 ; Flauss J.F. \& De Salvia M., La Convention européenne des droits de l'homme, Bruylant, Bruxelles, 1997 ; Maljean S., \& Dubois «La Convention européenne des droits de l'homme et le droit à l'information en matière d'environnement, $R G D I P$, 1998, p. 995; Marguenaud J.P., « Inventaire raisonné des arrêts de la Cour européenne des droits de l’homme relatifs à l'environnement », R.E.D.E., 1998, p.5-20 ; Kiss A., Conseil Européen du Droit de l'Environnement (CEDE), le droit à l'environnement, un droit fondamental dans l'Union européenne, Funchal, mars 2001, p. 25.
} 
pour les générations présentes et futures, de vivre dans un environnement propre à assurer leur santé et leur bien-être. Chaque partie devra garantir le droit d'accès à l'information, la participation du public dans le processus de prise de décision et d'accès à la justice en matière environnementale conformément aux dispositions de cette convention ». Dans le cadre de cette démarche, la nécessité de la protection de l'environnement a rencontré une certaine volonté d'asseoir un engagement ferme des États. L’article 37 de la Charte des droits fondamentaux de l’Union européenne de 2000 a porté sur la protection de l'environnement en ces termes :

«Un niveau élevé de protection de l'environnement et l'amélioration de sa qualité doivent être intégrés dans les politiques de l’Union et assurés conformément au principe du développement durable ».

Cette disposition est directement inspirée de l'Article 130 R 2 du Traité de Maastricht (intégration), des articles 2, 6 et 174 du Traité consolidé instituant la Communauté européenne et du préambule du Traité de l’Union européenne pour le principe du développement durable. De ce fait, la Charte n’ajoute rien aux textes existants de caractère obligatoire. En particulier, elle ne précise pas quels sont les droits des personnes et si elle énonce une obligation de protection de l'environnement, elle ne dit pas qui doit la mettre en œuvre ni quelle personne pourrait se plaindre en cas de non-respect. Selon Alexandre Kiss, une autre rédaction plus en rapport avec une Charte aurait été concevable. L’article 37 de la Charte est une régression car il ne reconnaît aucun droit aux citoyens contrairement à d'autres lois régionales comme en Afrique et en Amérique. La Charte ne constitue qu'une directive d’ordre politique adressée aux organes de l’Union elle-même. ${ }^{34}$

\section{OBLIGATIONS DES ÉTATS SIGNATAIRES À LA CHARTE AFRICAINE DES DROITS DE L'HOMME ET DES PEUPLES DANS LA PROTECTION ET LA PROMOTION DU DROIT À L'ENVIRONNEMENT}

En retenant comme principe le fait qu'il n’existe d'autre fondement à l'autorité de la règle de droit dans l'ordre international que la volonté étatique, les États signataires de la Charte Africaine des Droits de l'Homme et des Peuples ont volontairement souscrit au respect des

\footnotetext{
${ }^{34}$ Kiss A., op. cit. (n 33).
} 
obligations qui leur sont imposées par la Charte. ${ }^{35}$ Pour corroborer cette thèse, Claudia ScottiLam note que « Le propre de tout traité international est de présenter un caractère obligatoire pour les États contractants ». ${ }^{36}$ Certes, une obligation ne peut naître d’un traité pour un tiers que s’il l'accepte explicitement. Mais la création d'un droit est, en revanche, établie sur la base d'une présomption de consentement de sa part tant qu'il n'y a pas d'indication contraire. ${ }^{37}$ Deux dispositions de la Charte présentent manifestement ces obligations.

L’article 25 stipule que :

« Les États signataires de la présente Charte ont le devoir de promouvoir et d'assurer, par l'enseignement, l'éducation et la diffusion, le respect des droits et des libertés contenus dans la présente Charte, et de prendre des mesures en vue de veiller à ce que ces libertés et droits soient compris de même que les obligations et devoirs correspondants ».

L’article 26, quant à lui, prévoit que :

«Les signataires de la présente Charte ont le devoir de garantir l’indépendance des tribunaux et de permettre l'établissement et le perfectionnement d'institutions nationales appropriées chargées de la promotion et de la protection des droits et libertés garantis par la présente Charte ».

À la lecture de ces dispositions, il est important de noter que la protection de l'environnement implique différentes obligations et mesures à prendre $:^{38}$

Obligations négatives :

- $\quad$ Ne pas adopter de politiques ou de mesures portant atteinte à l'environnement ;

- Ne pas créer d'obstacles à l'acquisition, la diffusion ou l'utilisation des informations environnementales.

Obligations positives :

- Adopter un cadre juridique et administratif pour protéger l’environnement et éviter les pollutions ;

\footnotetext{
${ }^{35}$ Ruzié D. Droit international public : Mémentos, $16^{\text {ème }}$ éd., Dalloz, Paris, 2002, p. 281 ; Tchikaya B. Mémento

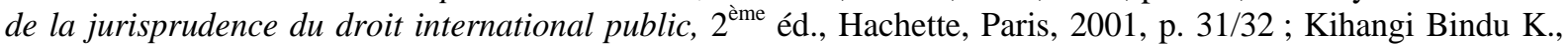
Droit International Public, Notes de cours, Université Libre des Pays des Grands Lacs, Inédit, 2010, p. 3.

${ }^{36}$ Sciotti-Lam C., L'applicabilité des traités internationaux relatifs aux droits de l'homme, Bruxelles, Bruylant, 2004, p .478.

${ }^{37}$ Articles 35 et 36 de la Convention de Vienne de 1965 sur le Droit des Traités du 23 mai 1969. Lire aussi

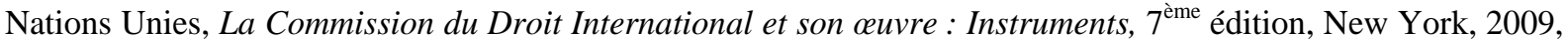
p. 137.

${ }^{38}$ Kiss A., op. cit. (n 33).
} 
- Adopter un cadre juridique pour recueillir, fournir, divulguer les informations environnementales ;

- Adopter un cadre juridique de surveillance de l'environnement et de détection des abus ;

- Adopter des procédures pour prendre en compte les avis des citoyens.

\section{Mesures :}

- Mise en œuvre des mesures concrètes de protection de l'environnement, de lutte contre la pollution et de restauration du milieu ambiant ;

- Surveillance des pollutions et inspections ;

- Génération et diffusion des informations environnementales ;

- $\quad$ Prise en compte des avis des citoyens ;

- Fonctionnement des organes de dialogue ;

- Exécution des décisions concernant la protection de l'environnement, sanctions et réparations des préjudices ;

- Ombudsman et tribunaux pour traiter les plaintes des citoyens pour atteinte à l'environnement.

En ce sens, pour concrétiser les obligations revenant aux États, la Cour de Justice de la Communauté Économique des États de l’Afrique de l’Ouest (CEDEAO) dans la décision Droits Économiques et Sociaux et Projet Responsabilité (SERAP) c. Nigéria, interpelle avec pertinence :

«... l’incapacité du gouvernement à promulguer des lois efficaces et à établir des institutions capables de réglementer les activités des entreprises, associée à son incapacité à traduire en justice les auteurs de la pollution, équivalait à une violation des obligations et engagements internationaux du Nigeria en matière de droits humains ». Elle a souligné que « la qualité de vie des personnes étant déterminée par la qualité de l’environnement, le gouvernement avait manqué à son devoir de maintenir un environnement satisfaisant et propice au développement de la région du delta du Niger ». ${ }^{39}$

En effet, la mise en place des institutions nationales pour la protection et la promotion des droits humains prévus par la Charte Africaine des Droits de l’Homme et des Peuples s’impose

\footnotetext{
${ }^{39}$ Amnesty International, la Cour de la CEDEAO ordonne au gouvernement du Nigeria de sanctionner les compagnies pétrolières responsables de pollution, titre récupéré le 14 mai 2013 sur : $<\underline{\text { http://www.amnesty.org/fr/for-media/press-releases/ground-breaking-ecowas-court-judgment-orders- }}$ government-punish-oil-companie>.
} 
aux États signataires. La création des cours et tribunaux reste une nécessité en vue de donner corps aux droits organisés par la Charte et garantir ainsi leurs caractère justiciable. Il serait aberrant de la part des juges de réserver une fin de non-recevoir à toute action judiciaire qui serait fondée sur la violation des droits humains organisés par la Charte, en l'occurrence le droit à un environnement sain.

Il est déplorable de noter que, malgré les engagements pris par les États, l’efficacité des instruments juridiques internationaux relatifs aux droits humains ne cesse de se heurter de plein fouet à l'intransigeance des souverainetés, particulièrement sur le continent Africain. ${ }^{40}$ Les États Africains demeurent jaloux de leur souveraineté et de leur indépendance, et ils considèrent avilissant de soumettre les problèmes internes à une tierce institution, fût-elle la Commission Africaine des Droits de l'Homme et des Peuples ou la Cour Africaine des Droits de l'Homme et des Peuples. L'expérience montre que dans l'ensemble, sur le continent africain, le droit à l'environnement qui est bien pourvu sur le plan juridique fait encore l'objet de certaines controverses. La difficulté de réparer les dommages écologiques, souvent très difficiles à évaluer à cause de leur nature, des conséquences à moyen et long terme qu’ils peuvent produire et surtout du caractère irréversible qu'ils peuvent revêtir, inquiètent largement. Sans sous-estimer la valeur du recours pour violation du droit à l'environnement, Kiss pense que l'on devrait ainsi insister sur les méthodes visant à prévenir ces violations. ${ }^{41}$ L'autre élément qui aurait pu protéger le droit à l'environnement en Afrique est l'information et la participation des populations en matière environnementale. Malheureusement, également à ce niveau, l’unanimité n'est pas au rendez-vous.

\section{LE PARTICULARISME DE LA CHARTE AFRICAINE ET DE LA JURISPRUDENCE DANS LE DOMAINE DES DROITS DES DEUXIÈME ET TROISIÈME GÉNÉRATIONS}

La conception africaine des droits humains reste marquée par un particularisme qui démontre l'attachement africain aux droits de seconde et troisième générations. La Charte Africaine organise des libertés collectives (association, réunion, participation politique) ainsi que les droits économiques, sociaux et culturels (DESC), notamment les droits au travail, à la santé et

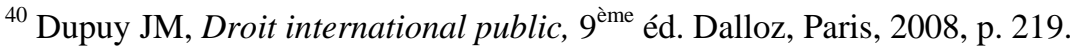

${ }^{41}$ Kiss A., Environnement, droits fondamentaux, droit international, droits fondamentaux, cahier du conseil constitutionnel $\mathrm{n}^{\circ}$ 15, titre récupéré le 9 octobre 2012 sur : <http://www.conseilconstitutionnel.fr/cahier/ccc15/env.htm>.
} 
à l'éducation. Les droits à l'auto-détermination, à la libre disposition des ressources naturelles, au développement et à un environnement sain y sont également affirmés. Les États membres doivent même protéger les populations locales des monopoles internationaux sur les ressources naturelles.

Il est fort remarquable que la nature progressive des DESC et de ceux de la solidarité alimente encore la discussion autour de leur caractère justiciable. Les DESC sont progressifs par nature et ils ne mettent à la charge des États que des obligations de moyens. En effet, la violation par un État du droit à l'eau, à l'alimentation, à l'éducation ou à la santé ne concerne généralement pas un individu isolé mais une partie importante de la population, a fortiori, dans des pays en développement aux infrastructures encore incomplètes. Afin d'assurer ces droits de manière satisfaisante, il est fondamental de développer les infrastructures appropriées et d'avoir pour ce faire les moyens humains, financiers et matériels nécessaires. Les ressources financières de l'État sont donc à tel point en jeu qu'on peut être amené à penser qu'il s'agit d'une question politique qui ne devrait pas faire l'objet d'une sanction par la branche du pouvoir judiciaire. Les droits collectifs font particulièrement l'objet de ces critiques et sont mêmes perçus parfois comme la somme d'autres droits. C'est ici qu'il faut présenter le caractère ô combien primordial de l'interdépendance et de l'indivisibilité des droits des trois générations. Les droits de la seconde et troisième générations sont à considérer comme une pré-condition à l'accomplissement des droits de la première génération. Par ailleurs, la violation des droits des trois générations est indéniablement liée. Par exemple, la pollution d'une rivière prive les individus de leur droit à un environnement sain, de leur droit d'accès à l'eau potable et, éventuellement, de leur droit à la dignité, voire à la vie. ${ }^{42}$ Suite à une dynamique interprétative, la Commission Africaine est arrivée à « judiciariser » les droits humains. ${ }^{43}$ Elle a ainsi adopté à la fois un principe et une méthode d'interprétation qui transcendent les catégories classiques des droits de l'homme. Le principe est celui de la justiciabilité de tous les droits reconnus par la Charte. ${ }^{44}$ C’est pourquoi, dans les cas du peuple Ogoni contre le Nigeria et Free Legal Assistance group contre le Zaïre, ${ }^{45}$ la commission saisit

\footnotetext{
${ }^{42}$ Serres F., Bardeche J., \& Fokoua N., Responsabilité sociale des entreprises : l'entreprise entre développement durable et droit au développement ou comment sortir de l'« éthique en toc » ? Titre récupéré le 9 octobre 2012 sur <http://www.droits-libertes.org/article.php3?id_article=159\#nb138>.

${ }^{43}$ Mutoy Mubiala, Le système régional africain de protection des droits de l'homme, Bruylant, Bruxelles, 2005, p. 89.

${ }^{44}$ Frouville O., L'intangibilité des droits de l'homme en droit international. Régime conventionnel des droits de l’homme et droit des traités, éd. Pedone, Paris, 2004 p. 261.

${ }^{45}$ Comm. ${ }^{\circ}$ 155/96, Social and Economic Rights Action Center, Center for Economic and Social Rights c/Nigeria, oct. 2001, $15^{\mathrm{e}}$ rapport annuel d'activités de la Commission africaine des droits de l'homme et des
} 
cette opportunité pour clarifier qu'il n’y a pas de droit dans la Charte Africaine que l'on ne puisse mettre en œuvre. La méthode consiste à expliciter pour chaque droit les « quatre niveaux d'obligations » qui incombent à l'État: l'obligation de respecter, de protéger, de promouvoir et de réaliser les droits. Appliquant la méthode au cas de l'espèce, la commission constate la violation du droit à la santé, du droit à l'environnement sain, du droit des peuples à la libre disposition de leurs ressources naturelles, et du droit au logement adéquat. Certes, l'autorité de la commission ne fait pas de ses décisions de véritables actes juridictionnels, ce que la Cour Africaine des droits de l'homme et des peuples est venue corriger. Dans l'affaire SERAP contre le Nigeria, ${ }^{46}$ la Cour de Justice de la CEDEAO a marqué un tournant décisif quant au caractère justiciable des droits des deuxième et troisième catégories garantis par la Charte Africaine des Droits de l'Homme et des Peuples. La Cour fait remarquer qu'il est bien établi que les droits garantis par la Charte Africaine sont opposables devant la Cour en citant l'article 9(4) du Protocole additionnel au traité établissant la Cour et l'article 4(g) du traité CEDEAO révisé, et cette dernière souligne sa compétence relative aux droits de l’homme prévus dans la Charte Africaine. La Cour a également jugé que le plaignant avait un intérêt à agir sur le fondement de l'action popularis qui permet à toute personne ou entité de contester la violation d'un droit public. Se fondant sur des jurisprudences étrangères, la Cour a ajouté que pour devoir agir dans un contentieux concernant le public, le plaignant doit démontrer qu'il a subi un dommage personnel ou qu'il possède un intérêt particulier qui doit être protégé. La Cour précise également que le demandeur doit démontrer qu’un intérêt public (collectif) digne d’être protégé a été violé et que ce droit est opposable. ${ }^{47}$

En ce qui concerne les droits largement reconnus dans les systèmes juridiques internes de la plupart des pays africains, la jurisprudence développée par la Cour Africaine des Droits de l'Homme et par la Cour de Justice de la CEDEAO pourrait servir de point de référence et d'inspiration puissant pour les juridictions internes, les gouvernements et les sociétés civiles des différents pays, au niveau de l'interprétation de ces droits. Il est ainsi rappelé à l'État d'adopter des mesures positives afin de prévenir les violations des droits des seconde et troisième générations. Par ailleurs, la Commission a retenu que l’État devrait enquêter sur les violations,

peuples, 2001-2002, Annexe 5 dans Frouville O., op. cit, p. 261. Affaire Free Legal Assistance group c. Zaïre, la Commission a conclu à la violation par l'État du droit à la santé physique et mentale et au principe de nondiscrimination.

${ }^{46}$ Droits Économiques et Sociaux et Projet Responsabilité (SERAP) c. Nigéria, 2012.

${ }^{47}$ Jurisprudences, affaires et autres décisions liées aux DESC, de la base de données jurisprudentielles du Réseau DESC, titre récupéré le 14 mai 2013 sur : <http://ebookbrowse.com/justice-desc-affaire-8-janvier-2010-pdfd278063504>. 
poursuivre les auteurs et permettre des recours aux victimes. Il s'agit du devoir de diligence des États développé par la jurisprudence dans le cadre des droits civils et politiques.

\section{LE DROIT À L'ENVIRONNEMENT DANS L'ORDRE JURIDIQUE CONGOLAIS}

En vue d'asseoir une culture démocratique de respect, de protection et de promotion des droits humains, l'incorporation d'un chapitre sur les droits humains dans une constitution a été annoncée depuis plusieurs décennies comme une nécessité majeure. Heynes Christof \& Viljoen Frans reconnaissent que :

"The domestic level is the most important level on which human rights should be protected by law in Africa. If the legal system of a particular country protects the human rights of everyone within its jurisdiction, there will be little or no need for higher levels of protection. Furthermore, without a certain level of respect for human rights in the participating domestic systems, a regional system, which is based on consensus and voluntary compliance by member states, cannot function" ${ }^{\text {, }}$.

Ainsi les États sont-ils généralement invités à incorporer ces instruments dans leurs ordres juridiques internes après la procédure de ratification. Cette procédure ne devrait se heurter à aucun obstacle si elle a lieu dans le respect du droit interne.

En effet, la consécration constitutionnelle du droit à l'environnement dans les États africains s'est faite un peu tardivement contrairement à certains États d'autres régions comme le Portugal (en 1976) ${ }^{49}$ et la Grèce. ${ }^{50}$ Ce n'est que depuis les années 1990 que l’on a assisté à un déferlement de consécrations du droit à l'environnement aussi bien sur le plan des textes fondamentaux des États que sur le plan des lois dans différents pays africains.

Dans cette dynamique, et pour répondre à ses obligations internationales, la RDC s'est engagée à veiller au respect, à la protection et à la promotion des droits de l'homme par sa loi fondamentale et des lois particulières. La Constitution du 18 février 2006 modifiée à ce jour par la Loi N 11/002 du 20 janvier 2011 est assez révélatrice au travers, notamment, de son

\footnotetext{
${ }^{48}$ Heynes C. \& Viljoen F., “The regional protection of human rights in Africa: An overview and evaluation” in Tiyambe Zeleza P \& McConnaughay PJ, Human rights, the rule of law, and development in Africa, University of Pennsylvania, USA Philadelphie, 2004, p. 131.

${ }^{49}$ La Constitution portugaise du 2 avril 1976 déclare dans son article 66 : « Chacun a droit à un environnement humain sain et écologiquement équilibré en même temps qu’il a le devoir de le défendre ».

${ }^{50}$ L'article 24 de la Constitution du 9 juin 1975 de la Grèce énonce un devoir de l’État : « La protection de l'environnement naturel et culturel constitue une obligation de l'État. L'État est tenu de prendre des mesures spéciales préventives ou répressives dans le but de sa conservation. »
} 
préambule et de son titre II portant sur les droits humains, les libertés fondamentales et les devoirs du citoyen et de l’État, en ces termes :

« Réaffirmant notre adhésion et notre attachement à la Déclaration Universelle des Droits de l'Homme, à la Charte Africaine des Droits de l'Homme et des Peuples, aux Conventions des Nations Unies sur les Droits de l'Enfant et sur les Droits de la Femme..., ainsi qu'aux instruments internationaux relatifs à la protection et à la promotion des droits humains ».

Les droits de la première, de la deuxième et de la troisième générations contenus dans la constitution présentent une innovation et une spécificité majeures de cette constitution du 18 février 2006 qui pose les jalons du constitutionnalisme et de la démocratie en RDC. ${ }^{51}$

Au moment de l’indépendance et pendant les années qui suivirent, les questions relatives à la reconnaissance des droits des deuxième et troisième générations n'étaient pas au cœur des débats des acteurs politiques. C'est ainsi que la quasi-totalité des textes constitutionnels qui ont régi le pays pendant la première et la deuxième République n’abordaient pas les questions notamment environnementales. Il a fallu attendre la ratification par la RDC de la Charte Africaine des Droits de l'Homme et des Peuples, et les changements intervenus au cours des années 1990, pour que les préoccupations environnementales aient un écho favorable dans les milieux politiques au pays. Seule la « révolution constitutionnelle » à partir des années 1990 a apporté un changement majeur dans le renforcement du déploiement constitutionnel des droits humains et dans la prise en compte des préoccupations environnementales.

Après la Constitution de Transition du 5 avril 2003, la Constitution du 18 février 2006 confirme le principe de la souveraineté permanente de l'État congolais sur ses ressources naturelles. ${ }^{52}$ Les ressources, constituant de richesses nationales, doivent faire l'objet d'une protection et d'une gestion rationnelle pour le bénéfice de toute la nation ${ }^{53}$ et dans le respect des règles environnementales. ${ }^{54} \mathrm{C}$ 'est dans cette philosophie que la Constitution rappelle que « Toute personne a droit à un environnement sain et propice à son épanouissement intégral. Elle a le devoir de le défendre. L’État veille à la protection de l'environnement et à la santé

\footnotetext{
${ }^{51}$ Les articles 11 à 33 portent sur les droits civils et politiques; les articles 34 à 49 portent sur les droits économiques, sociaux et culturels ; et les articles 50 à 61 portent sur les droits de la solidarité ou droits collectifs.

${ }^{52}$ Article 9 de la Constitution du 18 février 2006 de la RDC.

${ }^{53}$ Article 58 de la Constitution de la RDC du 18 février 2006 stipule que : « Tous les Congolais ont le droit de jouir des richesses nationales. L'État a le devoir de les redistribuer équitablement et de garantir le droit au développement.

${ }^{54}$ Article 123 point 15 de la Constitution de la RDC.
} 
des populations ${ }^{55}$. Les articles 47 et 48 de la Constitution portent respectivement sur les droits à la santé, à l’eau et à un logement décent. ${ }^{56}$

Le droit à l'environnement est ainsi considéré comme un droit fondamental de l'homme constitutionnellement garanti, protégé et justiciable. Son caractère procédural sous-entend le droit d'accès à l'information environnementale, le droit de participer au processus de prise de décision en matière d'environnement, le droit de recours devant les instances judicaires en cas de violation et le droit à la réparation. ${ }^{57}$ Le droit à l'environnement demeure ici un droit individuel et collectif. ${ }^{58} \mathrm{~A}$ ce titre, les associations représentatives des communautés locales et les organisations non gouvernementales nationales agréées et contribuant à la réalisation de la politique gouvernementale en matière environnementale peuvent exercer les droits reconnus à la partie civile en ce qui concerne les faits constituant une infraction aux dispositions de la loi et des mesures d'exécution, ou une atteinte, selon les accords et conventions internationaux ratifiés par la RDC et causant un préjudice direct ou indirect aux intérêts collectifs qu'elles ont pour objet de défendre. ${ }^{59}$ L'aspect substantiel contient le droit à l'eau, le droit à de meilleures conditions de vie et le droit au logement.

En effet, contrairement aux aspects procéduraux qui peuvent faire l'objet d'une application immédiate, cela n'est certes pas le cas pour les aspects substantiels du droit à l'environnement. Ces derniers obligent une démarche progressive ou graduelle. Tel est le cas

\footnotetext{
${ }^{55}$ Article 53 de la Constitution de la RDC.

${ }^{56}$ Article 47 « Le droit à la santé et à la sécurité alimentaire est garanti. La loi fixe les principes fondamentaux et les règles d'organisation de la santé publique et de la sécurité alimentaire. » Article 48 : « Le droit à un logement décent, le droit d'accès à l'eau potable et à l'énergie électrique sont garantis. La loi fixe les modalités d'exercice de ces droits ». Lire aussi l'article 47 de la Loi $N^{\circ}$ 11/009 du 9 juillet 2011 portant sur les principes fondamentaux relatifs à la protection de l'environnement 2011 qui stipule que :
}

« Toute personne a le droit de respirer un air qui ne nuise pas à sa santé.

Est interdite toute émission dans l'air susceptible d'incommoder la population ou de nuire à l'environnement et à la santé.

Les activités polluantes sont soumises soit au régime d'interdiction soit au régime d'autorisation préalable.

Un Décret délibéré en conseil des ministres fixe les normes d'émissions dans l'air ».

${ }^{57}$ Articles 8, 9, 10, 68 - 70 de la loi $N^{\circ} 11 / 009$ du 9 juillet 2011 portant sur les principes fondamentaux relatifs à la protection de l'environnement.

${ }^{58}$ Cette affirmation est confirmée par l'article 46 de la Loi ํํ 11/009 du 9 juillet 2011 portant sur les principes fondamentaux relatifs à la protection de l'environnement 2011 qui stipule que :

« Toute personne a droit à un environnement sain et propice à son épanouissement intégral.

Elle a le devoir de le défendre par toutes voies de droit en action individuelle ou collective. »

${ }^{59}$ Article 134 du Code Forestier 2002 de la République Démocratique du Congo. Lire aussi les articles 69 et suivants sur la responsabilité civile en matière environnementale de la loi $N^{\circ} 11 / 009$ du 9 juillet 2011 portant sur les principes fondamentaux relatifs à la protection de l'environnement. 
du droit au logement ou du droit à la santé. ${ }^{60} \mathrm{La}$ responsabilité civile en matière environnementale est aussi organisée par les articles 71 à 79 de la loi du 9 juillet 2011 portant sur les principes fondamentaux relatifs à la protection de l'environnement en RDC.

Par ce fait, la mise en œuvre du droit à l'environnement implique que chaque personne ait le droit d'intenter une action afin d'influer sur les décisions, actions ou omissions qui portent atteinte à l'environnement ou qui mettent en péril la vie de l’homme. Ces mesures sont au minimum l'accès à l'information environnementale, le droit de participer à certaines procédures de décision concernant l'environnement et, dans certains cas, le droit de recours devant diverses instances civiles ou administratives, le droit d'obtenir la cessation d'attaques à l'environnement lorsqu'elles sont illégales et le droit à la réparation des dommages causés. Cette dynamique législative interpelle tout esprit averti à jeter un regard critique sur son effectivité au travers des initiatives qui seraient prises par les cours et tribunaux en RDC dans le cadre de la protection et la promotion du droit à l'environnement.

\section{LES JURIDICTIONS CONGOLAISES FACE À LA PROTECTION ET À LA PROMOTION DU DROIT À UN ENVIRONNEMENT SAIN}

La Charte Africaine des droits de l'homme et des peuples a révolutionné la constitutionnalité et la culture des droits de l'homme dans un certain nombre de pays africains et la RDC ne fait pas exception. Il est du devoir des États signataires de veiller à l'applicabilité par les juges nationaux de la Charte qui désormais fait partie de leurs ordres juridiques. ${ }^{61}$

\section{Applicabilité de la Charte Africaine des Droits de l'Homme et des Peuples devant les cours et tribunaux congolais}

Étant d’une tradition moniste, la RDC reconnaît la primauté du droit international sur le droit interne. L’insertion formelle des traités internationaux en matière de droits de l’homme dans

\footnotetext{
${ }^{60}$ Smets H., « Une charte des droits fondamentaux sans droit à l'environnement », 4 Revue Européenne de droit de l'environnement 391, 2001.

${ }^{61}$ La question ne se pose pas dans la mesure où l'article 153 de la constitution confère aux juridictions de l'ordre judiciaire la compétence d'appliquer les traités internationaux dûment ratifiés qui sont placés au sommet de la hiérarchie normative. Dans cette optique, au Nigeria, une cour d'appel a décidé, en s’appuyant explicitement sur la CADHP, que les droits contenus dans cette charte étaient incorporés dans le droit nigérian. Tout Nigérian pouvait recourir à l'article 24 de la charte consacrant le droit à l'environnement pour exiger le respect dudit droit à son profit plutôt que de se fonder sur l'article 20 de la constitution qui n'était pas pertinent en l'espèce. SciottiLam C., op. cit. p .40.
} 
la constitution assure la conformité de l'ordre juridique interne par rapport à l'ordre juridique international. ${ }^{62}$ La RDC partage la théorie du « bloc de constitutionnalité » qui veut signifier que tous les traités auxquels la constitution fait référence dans son préambule ont une valeur constitutionnelle. C'est ainsi que les articles 215 et 153 de la Constitution de la RDC consacrent l'applicabilité directe des traités dans l’ordre interne congolais en leur donnant de surcroît prééminence sur le droit interne. ${ }^{63}$ Une jurisprudence congolaise permettant l'application directe des instruments juridiques internationaux est en phase d'élaboration notamment pour ce qui concerne le Statut de Rome. ${ }^{64}$

Le juge est habilité à appliquer les dispositions de la Charte Africaine des Droits de l'Homme et des Peuples qui n'établit aucune distinction entre catégories des droits. Cela est d'autant plus acceptable car il est admis que des normes ayant une simple valeur déclaratoire sur le plan international peuvent devenir contraignantes en droit interne qui leur accorde parfois même un rang constitutionnel ${ }^{65}$ pourvu que ces textes contiennent des dispositions claires, précises et juridiquement complètes. ${ }^{66}$ Claudia Sciotti-Lam ${ }^{67}$ « constate que dans les jeunes

\footnotetext{
${ }^{62}$ Cabinet du Président de la République Démocratique du Congo, Instruments internationaux relatifs aux droits de l'homme ratifiés par la République Démocratique du Congo, Journal Officiel de la République Démocratique du Congo, $40^{\text {ème }}$ année, numéro spécial, Kinshasa, 9 avril 1999, p. 1.

${ }^{63}$ La publication et la réciprocité sont des conditions de leur application dans l'ordre juridique interne et non de leur incorporation. Toutefois, ces deux conditions ne s'appliquent pas à l'égard des traités internationaux relatifs aux droits de l'homme. L'absence de publication d'un traité international relatif aux droits de l'homme ne devrait pas empêcher son application dans l'intérêt des individus. Qu'il s'agisse des traités universels ou régionaux, le régime des traités en droit congolais RDC est organisé par deux articles importants de la Constitution. L'article 215 stipule que « Les traités et accords internationaux régulièrement conclus ont dès leur publication, une autorité supérieure à celle des lois ». L'article 153 al. 4 ajoute que " Les cours et tribunaux, civils et militaires, appliquent les traités internationaux dûment ratifiés, les lois, les actes réglementaires pour autant qu'ils soient conformes aux lois, ainsi que la coutume pour autant que celle-ci ne soit pas contraire à l'ordre public et aux bonnes mœurs ». Dupuy JM, op. cit. p. 224 ; Nobirabo Musafiri P., Droit foncier des peuples autochtones et le droit international : cas des peuples de la forêt «Pygmées » de la RD du Congo, Stämpfli Éditions Berne, 2007, p. 401 - 419. Mazyambo Makengo A., op. cit. p. 243 ; Ministère Public et Partie Civile contre SLt Baseme Ofidi et Autres, Tribunal Militaire de Garnison de Goma RP 356/2009 RMP 0042/KNG/09, 19 et suiv. 24 avril 2009 (République Démocratique du Congo), titre récupéré le 4 avril 2009 sur : <www.justice.gov.cd $>$
}

64 RMP 154/PEN/SHOF/05 - RP 084/2005, titre récupéré le 27 août 2012 sur : $<$ http://www.rwandadocumentsproject.net/gsdl/collect/drc/index/assoc/HASHa9ef.dir/18DRCSectionIIIChapter Two.pdf visité le 27/8/2012>. Lire Lifumba Waka (RMP 064/AMS/SHP.06) ; Kilwa (RMP 0064/NMB/2005 RP 010/2006). Jugement RP 356/2009 (RMP 0042/KNG/09) en date du 24 avril 2009, le Tribunal Militaire de Garnison de Goma statuant au premier degré en matière répressive, dans la juridiction de WALIKALE; Jugement de la Cour Militaire de la Province Orientale, RPA No 003/2007, RP $N^{\circ} 101 / 2006$, RMP N $^{\circ}$ 545/PEN/2006 faisant directement référence au Décret-loi No 003/2002 du 30 mars 2002 sur la Ratification du Statut de Rome.

${ }^{65}$ Sciotti-Lam C., op. cit. p. 253.

${ }^{66}$ Mazyambo Makengo Kisisala A., op. cit. p. 244. De Vries Reilingh J., L'application des pactes des nations unies relatifs aux droits de l'homme de 1966 par les cours constitutionnelles ou par les cours suprêmes en Suisse, en Allemagne et au Canada, Bruxelles, Bruylant, 1998, p. 176.

${ }^{67}$ Sciotti-Lam C., op.cit. p. 253. 
démocraties, le droit interne ne fait pas la distinction entre les différentes sources du droit international des droits de l'homme. Celles-ci s'imposent en droit interne, quelle que soit leur origine, interne ou internationale, ou leur nature, obligatoire ou déclaratoire. Ce constat est fondé notamment sur le rôle de la déclaration universelle des droits de l'homme dans les constitutions ». Pour sa part, Maurice Kamto ${ }^{68}$ reconnaît qu’ « en raison de la constitutionnalisation des droits, leur garantie juridictionnelle [...] se fait, d'une part, par le biais du contrôle de la constitutionnalité des lois et d'autre part, par le biais du contentieux des droits et libertés ». La situation se présente clairement de cette manière en RDC où pour le droit à l'environnement, comme tous les autres droits ayant une valeur constitutionnelle, l'action en inconstitutionnalité est permise aux administrés en cas de violation d'obligations qui en découlent. Toute personne a désormais le droit de saisir la Cour Constitutionnelle non seulement par voie d'exception mais aussi par voie d'action. ${ }^{69}$ Le contrôle a priori prévu par l'article 160 de la Constitution congolaise permet une protection préventive alors que le contrôle a posteriori prévu aux articles 161 et 162 de la Constitution permet la réparation des violations des droits garantis. Évidemment, concevoir un texte, l'écrire et l'édicter, c'est une chose, l'appliquer sur le terrain à des situations concrètes en est une autre. Il en découle un décalage énorme entre ce qui doit être et ce qui est fait en réalité. La règle de droit et la loi formulent et imposent des idéaux avec comme objectif de les faire appliquer par les juridictions. ${ }^{70}$ La question se pose donc de savoir si les magistrats congolais font allusion dans leurs jugements aux instruments internationaux relatifs aux droits de l'homme ratifiés par la RDC, particulièrement la Charte Africaine des Droits de l'Homme et des Peuples. Jean Didier Boukougou, cité par Laurence Hervé Etoa, pense à ce sujet que l'application de la Charte Africaine des Droits de l'Homme et des Peuples, comme d'ailleurs des autres instruments régionaux et internationaux relatifs aux droits de l'homme, ne suscite aucun enthousiasme de la part des autorités d'Afrique centrale. Cette position est celle de beaucoup d'autres auteurs

\footnotetext{
${ }^{68}$ Kamto M., « Charte africaine, instruments internationaux de protection des droits de l'homme, constitutions nationales : articulations respectives », dans Flauss J.F. et Lambert - Abdelgawad E., (S/dir.), L'application nationale de la charte africaine des droits de l'homme et des peuples, Bruxelles, Bruylant, 2004 p. 37-38; Nyaluma Mulagano A., La prise en charge de l'enseignement de base par les parents en RDC : les recours possibles, p. 13/14. Titre récupéré le 6 octobre 2012 sur : <http://www.google.co.uk/search?q=LA\%20PRISE\%20EN\%20CHARGE\%20DE\%20L\%27ENSEIGNEMENT \%20DE\%20BASE\%20PAR\%20LES\%20PARENTS\%20EN\%20R.D.C.\%20\%3A\%20LES\%20RECOURS\%20 POSSIBLES\%20Par\%20Arnold\%20NYALUMA\%20MULAGANO\%20*\&ie=utf-8\&oe=utf$8 \& a q=t \& r l s=$ org.mozilla:fr:official\&client=firefox-a\&source=hp\&channel=np $>$.

${ }^{69}$ Article 162 de la Constitution du 18 février 2006 de la RDC.

${ }^{70}$ Kihangi Bindu K., « De l’applicabilité des traités internationaux relatifs aux droits de l’homme ratifiés par la République Démocratique du Congo ", Analyste Topic, № 6, Université Libre des Pays des Grands Lacs, 2012, p. 68.
} 
qui pensent que la Charte Africaine n'est qu'une phase. C'est le cas de Sikondo M-H et Gabou A. qui pensent que la Charte Africaine des Droits de l'Homme et des Peuples « est un cas typique de comédie solennelle que les États africains utilisent comme simple habit de gala pour mondanités internationales ${ }^{71}{ }^{71}$ Dans l'ensemble, l'Afrique a franchi un pas de géant en présentant clairement une construction théorique pour abriter le droit à l'environnement qui malheureusement fait l'objet de nombreuses insuffisances dans sa phase de réalisation. Cela semble être le cas en RDC où il n’est pas évident que les avocats ou les justiciables invoquent directement ce droit avec succès devant les cours et tribunaux. Existe-t-il une certaine jurisprudence congolaise en la matière, particulièrement en matière de droit à un environnement sain?

\section{La jurisprudence congolaise en matière de droit de l'homme à un environnement sain}

En tant que droit constitutionnellement garanti en RDC, le droit à l'environnement sain peut faire l'objet d'une action en justice individuelle et/ou collective. ${ }^{72}$ Le juge a le devoir de garantir ce droit ${ }^{73}$ et d'appliquer directement les dispositions de la Charte Africaine des Droits de l’Homme et des Peuples. Les juridictions congolaises se doivent de motiver leurs décisions non seulement au regard du droit interne mais aussi au regard du droit international. Les juridictions internes peuvent se référer aux textes internationaux ainsi qu'à la jurisprudence qui leur est propre avec des conceptions de droit interne. Il est déplorable de noter une forte hésitation au sein des juges congolais quant au caractère justiciable du droit à un environnement sain en RDC malgré sa consécration constitutionnelle et la jurisprudence disponible sur le plan continental. ${ }^{74}$ Cette jurisprudence a été fortement renforcée par la Cour

\footnotetext{
${ }^{71}$ Jiatsa Meli H., op. cit.

${ }^{72}$ Article 46 de la Loi de juillet 2011

« Toute personne a droit à un environnement sain et propice à son épanouissement intégral.

Elle a le devoir de le défendre, par toutes voies de droit en action individuelle ou collective. »

${ }^{73}$ Article 150 de la Constitution du 18 février 2006 de la RDC.

${ }^{74}$ Le comportement du juge du Tribunal de Grande Instance de Bukavu qui a tenté de produire une réflexion bien que de façon timide quant à l'importance des droits de l'homme consacrés par la Charte Africaine des Droits de l'Homme et des Peuples mérite toute notre attention. Dans son argumentaire, le demandeur présente clairement la Charte Africaine des Droits de l'Homme et des Peuples comme un instrument faisant partie intégrante du droit positif congolais. Les droits qu'elle consacre peuvent faire l'objet d'une réclamation devant les instances judiciaires en RDC. Sans questionner le caractère de justiciabilité de ces droits, le juge répond en ces termes :
}

« Il en découle que le droit à l'égalité et à la non-discrimination garanti par les instruments juridiques internationaux... et par la Constitution de la République Démocratique du Congo n’ont pas été violés par les défendeurs. Il en est de même du droit d'accès à l'énergie électrique pour la 
de Justice de la CEDEAO dans l'affaire SERAP contre le Nigeria dans laquelle elle souligne que le Nigeria devrait être tenu responsable pour avoir violé les articles 21 (sur la disposition des peuples de leurs richesses et de leurs ressources naturelles) et 24 (sur le droit à un environnement satisfaisant) de la Charte Africaine des droits de l'homme et des peuples en ne protégeant pas le delta du Niger et ses habitants des opérations des compagnies pétrolières qui pendant de nombreuses années ont dévasté la région. Selon la Cour, le droit à l'alimentation et à la vie sociale des peuples du delta du Niger a été violé par la détérioration de leur environnement, détruisant ainsi leur possibilité de gagner leur vie et de jouir d'un niveau de vie sain et suffisant.

Les cas camerounais et Sud-Africain ${ }^{75}$ bénéficient de tous nos suffrages quant à la justiciabilité du droit à un environnement sain étant entendu que la reconnaissance d’un droit implique ipso facto la possibilité d’un recours dans le cas de sa violation. Le 16 août 1975, dans un arrêt de la cour d’appel de Yaoundé concernant un litige opposant la société Paterson Zochonis à Atangana Protais, le droit à l'environnement était revendiqué. Certes à ce niveau, le juge n’était pas conscient que son verdict protégeait le droit à l'environnement, car le problème de fond n'était pas le droit à un environnement sain mais plutôt d'un individu qui accusait une société d’avoir répandu des mauvaises odeurs, des émanations putrides ou des fumées nocives. Dans l'esprit du juge de l'époque, c’était bien plus le code civil qu'il fallait appliquer, puisque dans le cas présent, il s’agissait d'un trouble anormal de voisinage. Dans ce cas, le droit à l’environnement n’a été protégé que de manière incidente. De même, en 1983, dans un arrêt du 12 octobre, le Tribunal de Grande Instance de Yaoundé a travaillé sur l'affaire opposant Nkouedjin Yonda à la société Exarcos. Également dans ce cas, le juge a protégé le droit à l'environnement en sanctionnant la société de travaux publics dont les activités avaient fait stagner les eaux de pluies à l'entrée de la maison d'un individu. ${ }^{76}$ Évidemment, le juge n'avait pas à l'esprit le besoin de protéger le droit à l'environnement en tant que droit de l’homme, mais plutôt de protéger le droit de propriété et de régler des

bonne et simple raison que depuis la conclusion de son abonnement en décembre 2006 jusqu’à la saisine du Tribunal de Céans, le demandeur est pourvu en énergie électrique. » Jugement du Tribunal de Grande Instance de Bukavu, R.C. 7206 du 14/07/2008.

${ }^{75}$ Dans un certain nombre des cas, les juridictions sud-africaines ont été amenées à prendre des actions proactives pour la protection de l'environnement : Minister of Health and Welfare $v$ Woodcarb (Pty) Ltd (1996) (3) SA 155 (N); Van Huyssteen v Minister of Environmental Affairs and Tourism (1996) (1) SA 283 (C) ; Die Vereniging van Advokate v Moskeeplein (Edms) Bpk (1982) (3) SA 159 (T). Kihangi Bindu K., Environmental and developmental rights in the Southern African Development Community: Specific reference to the Democratic Republic of Congo and the Republic of South Africa, op. cit. p. 280.

${ }^{76}$ Jiatsa Meli H., op. cit. 
troubles anormaux de voisinage entre voisins. Le droit à un environnement sain est protégé mais de manière indirecte et par le canal d'autres droits. Pourtant, la constitution offre la possibilité de revendiquer un cadre de vie sain sans recourir à aucun autre droit. Si le droit à un environnement sain existe par lui-même, pourquoi ne devrait-il pas aussi exister par luimême devant le juge ? Ce droit gagnerait à se voir conférer un statut qui lui permettrait par lui-même d'exister devant le juge. Ainsi suivra la possibilité pour les personnes physiques de se faire dédommager lorsque leur droit à un environnement sain a été violé. Car, pour que le droit à l'environnement soit effectif, sa violation doit pouvoir être réparée. La revendication d'un droit à l'environnement peut être aussi formulée par une personne morale (État, ONG et association à but non lucratif). ${ }^{77}$ Ainsi en RDC, les ONG et associations à but non lucratif peuvent saisir le juge lorsque le droit à un environnement sain a été violé. Elles ne peuvent pas seulement se limiter à dénoncer l'atteinte au droit à l'environnement devant le procureur ou les autorités de police judiciaire pour lancer la procédure judiciaire. Elles peuvent également exercer les droits reconnus à la partie civile en ce qui concernent les faits constituant une infraction au regard de la législation congolaise en la matière. Le caractère collectif prend juridiquement corps dans un procès où le droit à l'environnement est en cause. ${ }^{78}$ Les conditions générales pour intenter en justice qui sont la capacité, l’intérêt et la qualité ne les excluent pas pour ne pas saisir le juge. Il s'agit d'une évolution impressionnante du droit congolais pour le respect, la protection et la promotion du droit à un environnement sain. Des actions en justice peuvent être intentées pour obtenir la sanction administrative, pénale ou civile contre les atteintes aux éléments de l'environnement. Il est dommage de noter que malgré cette applicabilité permise par les textes, la réalité sur le terrain demeure inquiétante car le droit à l’environnement ne s’applique pas avec la même énergie devant le juge congolais. Le contentieux environnemental est quasi inexistant devant les juridictions congolaises malgré les cas de violations presque quotidiennes et manifestes de ce droit et de la

\footnotetext{
${ }^{77}$ Ici l’État se manifeste sur le terrain par l'administration qui s’est illustrée comme véritable défenseur du droit à l'environnement devant le juge. L’administration porte les sociétés industrielles devant le juge pour violation du droit des populations à un environnement sain. En agissant ainsi, on affirme le caractère collectif du droit à l'environnement. La procédure tarde parfois lorsque l'administration et l'entreprise qui a porté atteinte à l'environnement ne parviennent pas à un accord. Sur le plan pénal de l'environnement, l'État est aussi très régulièrement intervenu pour porter plainte contre les personnes allant à l'encontre du droit à l'environnement, en chassant, en tuant ou en domestiquant les espèces en violation de la réglementation en vigueur. Dans ce dernier cas, une fois de plus, la protection du droit à l'environnement est indirecte car ici le droit à l'environnement n'est retenu qu'à travers la protection de certaines espèces.

${ }^{78}$ Article 134 du Code Forestier de 2002. Kihangi Bindu K., «The environmental law framework of the Democratic Republic of Congo and the balancing of environmental and developmental interests» in Michael Faure M \& Du Plessis W., (S/dir.) The balancing of interests in environmental law in Africa, Pretoria University Law Press, University of Pretoria, South Africa, 2011, p. 105.
} 
législation en matière environnementale en général. Cela est d'autant plus vrai lorsque l'on constate combien la dégradation de l'environnement et l'absence de toute politique cohérente environnementale en RDC sont déplorables. La jurisprudence en RDC n'a donc pas encore contribué à la définition notamment des régimes de protection de l'environnement, de l'étendue des droits environnementaux des citoyens, des obligations de l'État, etc. Le besoin est encore énorme en ce domaine. Cet état des choses en RDC mérite une attention particulière du gouvernement congolais et de la communauté internationale dans son ensemble avant qu'il ne soit trop tard. C'est pourquoi nous ne cessons de militer pour la mise en place d'une juridiction en matière environnementale en RDC, pour le renforcement des compétences des juges et pour une expertise éclairée en ce domaine. ${ }^{79}$ Il est bien connu que les problèmes environnementaux se posent en termes globaux car les menaces écologiques ignorent les frontières de souveraineté érigées par les États. ${ }^{80}$ Ainsi, face au risque de propagation transfrontalière, l'idée d'une ingérence verte est en train de faire son chemin. Simple phénomène ponctuel à la suite de la notion d'ingérence humanitaire, la question mérite une attention particulière en Afrique car bon nombre de pays de ce continent pourraient être des victimes potentielles d'une telle ingérence. Cette approche peut déboucher sur une nouvelle forme de domination des bailleurs de fonds (institutions ou gouvernements) dans la prise de décisions environnementales. Allons-nous donc vers une marginalisation verte de l’Afrique pour qui le mécanisme d'échange de la dette contre la protection de l'environnement a été guère probant ? $^{81}$

\footnotetext{
79 "Independent specific courts or tribunals dealing with environmental matters must also be established. It has been proved that the 'uniqueness of jurisdiction' which characterizes the Congolese judicial system does not allow judges to specialize in environmental jurisprudence. Judgments offered by the courts or tribunals run the risk of becoming approximate. In addition, judges seem to be unaware of the provisions of the international instruments ratified by the DRC on human rights 45 and do not give close attention to the human rights guaranteed by the Constitution. An important campaign to train judges in the DRC needs to be carried out because courts and tribunals are the custodians of human rights (Article 150). The jurisprudence on human rights will be helpful in fleshing out the content of environmental and development rights and will guide parties in taking any human rights claim to court. Parliament is still playing a pioneering role by providing the necessary and requested legislation to support those various human rights in the light of the Constitution". Lire Kihangi Bindu K., Environmental and developmental rights in the SADC: Specific reference to the Democratic Republic of Congo and the Republic of South Africa, LAP, Lambert Academic Publishing, Germany, 2011, p. $340 / 341$.

${ }^{80}$ Kihangi Bindu K., "Environmental legal requirements and the exploitation of natural resources in a post conflict country: A case study of the Democratic Republic of Congo", The A38 Journal of International Law, Vol. 1, Édition 3, 2012, p. 1-5.

${ }^{81}$ Dans ses analyses, Kamto M. note que «comme l'ingérence humanitaire en Droit International Public, la politique de «l'ingérence verte » ou le "droit d'ingérence écologique » se résume par la faculté pour un ou plusieurs États de pouvoir s'immiscer, sans en être requis, sur le territoire d'un autre État pour protéger ou restaurer une partie du patrimoine commun détruit ou en danger de destruction. Bien qu'elle soit partie de considérations apparemment généreuses de solidarité écologique, l'ingérence verte ainsi entendue ne peut que susciter des réserves. Certes, sa mise en œuvre pourrait permettre de lutter contre certaines formes de
} 


\section{CONCLUSION ET RECOMMANDATIONS}

La Charte Africaine des Droits de l'Homme et des Peuples est un instrument juridique de taille qui a supposé un bouleversement dans l'approche de plusieurs acteurs notamment étatiques. La jurisprudence disponible sur le plan continental démontre suffisamment le caractère justiciable du droit à l'environnement. On peut désormais affirmer qu'il s’agit d'un droit bénéficiant d'une protection égale aux autres droits humains reconnus à la fois sur les plans national et international. Les cours et tribunaux ont la responsabilité de le faire respecter, de le protéger et de le promouvoir. La ratification de la Charte Africaine des Droits de l'Homme et des Peuples par la RDC et l'évolution constitutionnelle à partir de 1990 en RDC sont des aspects assez révélateurs et impressionnants à ce sujet. Les événements des années 1990 ont constitué une pépinière significative pour la reconnaissance constitutionnelle du droit à un environnement sain, le devoir de toute personne de le défendre, et à l’État de veiller à la protection de l'environnement et à la santé des populations. Le pouvoir judiciaire doit exercer son rôle de garant de ce droit qui peut faire l'objet d'une action à la fois individuelle et collective. Cependant, il est déplorable de noter une certaine nonchalance de la part du juge congolais qui semble rester en marge de l'évolution constitutionnelle et législative applicable. Il convient ici de rappeler que les hésitations du juge et la quasi absence de litiges en matière environnementale, particulièrement une lecture judiciaire du droit à l'environnement, sont dues à plusieurs raisons notamment :

- L'absence d'une expertise judiciaire en matière environnementale ;

- L'absence d'un tribunal en matière environnementale ;

- L’ignorance par la population de ses droits et des mécanismes de revendication ;

- L’ignorance de certains magistrats qui hésitent à s'engager dans un procès où le droit à l'environnement est en cause faute de manque d'une expertise conséquente ;

coopération qui conduisent à une exploitation effrénée et écologiquement irrationnelle des ressources naturelles dans certains pays africains. Cependant, il y a lieu de faire remarquer que cette sorte « d'assistance à nature en danger » risque de porter en elle ce que certains qualifieraient de " cache sexe », d'une sorte d'impérialisme écologique. On peut craindre en effet que le «droit d'ingérence verte » ne transforme le Sud, et tout particulièrement l'Afrique, en une « immense arrière-cour écologique des pays occidentaux, en une réserve où ils n’hésiteront pas à intervenir pour redresser des gouvernements ou des peuples «braconniers » dont les délits s’apprécieront à la hauteur de leur réticence à apporter, par une application prompte et aveugle sur leur territoire, la légitimité qui fait tant défaut aux solutions que la "Société internationale » propose pour résorber la crise écologique. Le danger imminent de voir institutionnaliser une espèce de « gendarmerie écologique mondiale » qui érigerait les pollueurs en gardiens du temple et condamnerait les seuls pays pauvres pour délinquance écologique. L’Afrique post-coloniale est appelée à agir et à se tenir convenablement debout sur plusieurs fronts à la fois, ici et maintenant. » Kamto M. Droit de l'environnement en Afrique, Edicef, Paris, 1996, p. 60. 
- La mauvaise gouvernance qui ne favorise pas le respect des besoins élémentaires de la population (logement, éducation, soins de santé, eaux et énergie,...) ; et

- $\quad$ L’absence d'une véritable culture des droits de l'homme.

Pour une applicabilité effective de la Charte Africaine des Droits de l'Homme et des Peuples particulièrement, certaines conditions préalables doivent être réunies :

- L’instauration d'une véritable culture démocratique en RDC et la mise en place d'un programme d'éducation environnementale ;

- La création d'un tribunal en matière environnementale et le renforcement des capacités des juges dans ce domaine ;

- L’instauration d'un État de droit qui se plie devant le droit ;

- L'organisation de séances d'éducation et d'information de la population sur les droits de l'homme, en particulier le droit à un environnement sain ;

- La création d'autres institutions indépendantes de soutien à la démocratie comme l'observatoire des droits humains ou une institution de médiateurs ou d'ombudsman.

L'absence d'une jurisprudence efficace en matière environnementale, et des droits de l'homme en général, doit désormais interpeller les chercheurs qui doivent sortir de leur isolement et éclairer la lanterne des uns et des autres. Le monisme adopté par la RDC doit servir de point de repère au juge congolais qui doit faire prévaloir les intérêts du peuple en appliquant directement la Charte Africaine des Droits de l'Homme et des Peuples chaque fois qu'il est sollicité. L’acteur politique congolais ainsi que les magistrats sont appelés à surmonter leurs hésitations qui affaiblissent les revendications et actions tendant au respect, à la protection et à la promotion du droit à un environnement sain consacré par la Charte, par d'autres instruments juridiques internationaux ratifiés par la RDC, et par la Constitution du 18 février 2006. La démocratie constitutionnelle tant chantée en RDC pourra ainsi devenir une réalité au-delà des simples paroles. 


\section{BIBLIOGRAPHIE FINALE}

Ankumah Everlyne A., The African Commission on Human and Peoples' Rights: Practice and procedures Vol. 16, Martinus Nijhoff Publishers, The Hague, London, Boston, 1996.

Boyle A., \& Anderson M., Human Rights Approaches to Environmental Protection, Clarendon, Oxford, 1998.

Brown Weiss E., (ed.) Environmental change and international law: New challenges and dimensions, Tokyo, United University Press, 1992.

De Vries Reilingh J., L'application des pactes des nations unies relatifs aux droits de l'homme de 1966 par les cours constitutionnelles ou par les cours suprêmes en Suisse, en Allemagne et au Canada, Bruxelles, Bruylant, 1998.

Déjeant - Pons M., « Le droit de l'homme à l'environnement : Droit fondamental au niveau européen dans le cadre du Conseil de l’Europe”, R.J.E., N²4, 1994.

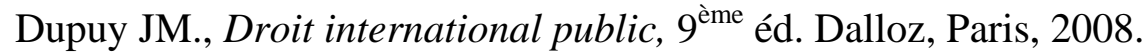

Faure M. \& Du Plessis W., (Dir.) The balancing of interests in environmental law in Africa, Pretoria University Law Press, University of Pretoria, South Africa, 2011.

Flauss J.F. \& De Salvia M., La Convention européenne des droits de l'homme, Bruylant, Bruxelles, 1997.

Flauss J.F., \& Lambert - Abdelgawad E., (Dir.), L'application nationale de la charte africaine des droits de l'homme et des peuples, Bruxelles, Bruylant, 2004.

Frouville O., L'intangibilité des droits de l'homme en droit international. Régime conventionnel des droits de l’homme et droit des traités, éd. Pedone, Paris, 2004.

Kamto M. Droit de l'environnement en Afrique, Edicef, Paris, 1996.

Kihangi Bindu K., “De l’applicabilité des traités internationaux relatifs aux droits de l’homme ratifiés par la République Démocratique du Congo », Analyste Topic, №6, Université Libre des Pays des Grands Lacs, 2012.

— "Environmental legal requirements and the exploitation of natural resources in a post conflict country: A case study of the Democratic Republic of Congo”, The A38 Journal of International Law, Vol. 1, Edition 3, 2012. 
- Environmental and developmental rights in the Southern African Development Community: Specific reference to the Democratic Republic of Congo and the Republic of South Africa, Lap Lambert Academic Publishing, Germany, 2011.

Kiss A., \& Shelton D., Guide to international environmental law, Martinus Nijhoff Publishers, Leiden/Boston, 2007.

Kiss A., Conseil Européen du Droit de L’Environnement (CEDE), le droit à l'environnement, un droit fondamental dans l’Union Européenne, Funchal, mars 2001.

— Environnement droits fondamentaux, droit international, droits fondamentaux, Cahier du conseil constitutionnel $n^{\circ} 15$.

Ksentini Z., Droit de l'Homme et Environnement, Commission des droits de l'Homme, Conseil économiques et social des Nations Unies, 6 juillet 1994.

Maljean S., \& Dubois «La Convention européenne des droits de l'homme et le droit à l'information en matière d'environnement, RGDIP, 1998.

Marguenaud J.P., "Inventaire raisonné des arrêts de la Cour européenne des droits de l’homme relatifs à l'environnement, R.E.D.E., 1998.

Mekouar A. M., Le droit de l'environnement dans la Charte Africaine des droits de l'homme et des peuples, étude juridique de la FAO, avril 2001.

Mutoy Mubiala, Le système régional africain de protection des droits de l'homme, Bruylant, Bruxelles, 2005.

Nations Unies, La Commission du Droit International et son ouvre: Instruments, $7^{\text {ème }}$ édition, New York, 2009.

Nobirabo Musafiri P., Droit foncier des peuples autochtones et le droit international : Cas des peuples de la forêt « Pygmées » de la RD Congo, Stämpfli Editions, Berne, 2007.

Prieur M., Droit international et comparé de l'environnement : Les principes généraux du droit de L'environnement, cours $\mathrm{n}^{\circ} 5$, formation a distance, campus numérique, Université de Limoges.

Programme des Nations Unies pour le Développement, Projet Appui aux institutions de la transition «Mandats, rôle et fonctions des pouvoirs constitués dans le nouveau système politique de la République Démocratique du Congo : Journées d’information et de formation 
organisées à l'intention des parlementaires, des députés provinciaux et de hauts cadres de l’administration », Kinshasa, 2007.

Ruzié D., Droit international public : Mémentos, 16 ème éd., Dalloz, Paris, 2002.

Sciotti-Lam C., L'applicabilité des traités internationaux relatifs aux droits de l'homme, Bruxelles, Bruylant, 2004.

Sélection des documents clés de l'Union Africaine relatifs aux droits de l'homme, Pretoria University Law Press, Pretoria, 2008.

Serres F., Bardeche J., \& Fokoua N., Responsabilité sociale des entreprises : l'entreprise entre développement durable et droit au développement ou comment sortir de l' « éthique en toc ».

Smets H., «Une charte des droits fondamentaux sans droit a l'environnement » 4 Revue Européenne de droit de l'environnement 2001.

Sudre F., Droit international européen des droits de l’homme, $5^{\text {ème }}$ éd., PUF, Paris, 1989.

Tchikaya B. Mémento de la jurisprudence du droit international public, $2^{\text {ème }}$ éd., Hachette, Paris, 2001.

Thornton J. \& Beckwith S., Environmental law, $2^{\text {nd }}$ ed. Sweet and Maxwell Thomson London, 2004.

Tiyambe Zeleza P \& McConnaughay PJ, Human rights, the rule of law, and development in Africa, University of Pennsylvania, USA Philadelphia, 2004. 